\title{
Superiority of raw biomass and potassium hydroxide in preparation of ultrahigh nitrogen doping of carbon for $\mathrm{NH}_{3}-\mathrm{SCR}$ reaction
}

\author{
Qiaoyan $\mathrm{Li}^{\dagger *}$, Yaqin $\mathrm{Hou}^{* \dagger}$, Jiancheng Wang ${ }^{\S}$, Yongjin Liu ${ }^{\dagger \dagger}$, Ning Xiang ${ }^{\dagger \star}$, Zhanggen Huang $*^{\dagger}$ \\ ${ }^{\dagger}$ State Key Laboratory of Coal Conversion, Institute of Coal Chemistry, Chinese Academy of Sciences, \\ Taiyuan 030001, PR China \\ $\$$ University of Chinese Academy of Sciences, Beijing 100049, PR China \\ $\S$ Key Laboratory of Coal Science and Technology, Ministry of Education and Shanxi Province, Taiyuan \\ University of Technology, Taiyuan, 030024, PR China
}

To whom correspondence should be addressed:

*Phone: +86-0351-4043727. E-mail: zghuang@sxicc.ac.cn (Z.G.).

E-mail: houyaqin@sxicc.ac.cn (Y.H.).

\section{Nine pages \\ Four figures \\ Four tables}




\section{Characterization Techniques}

Elemental analyses $(\mathrm{C}, \mathrm{O}, \mathrm{N})$ were conducted on a Vario Micro elemental analys is instrument from Vario EL CUBE. The special surface area and pore size distribution were measured by the $\mathrm{N}_{2}$ adsorption-desorption on Quantachrome Autosorb II at $77 \mathrm{~K}$. The chemical states of elements were characterized by X-ray photoe lectron spectroscopy (XPS), which were recorded on AXIS ULTRA DLD spectrometer with $\mathrm{Al} \mathrm{K \alpha} \mathrm{X}$-ray exciting radiation. The crystalline structure of the samples was investigated by a Bruker D8 diffractometer $(40 \mathrm{kV}, 100 \mathrm{~mA}, \mathrm{Cu} \mathrm{K \alpha}, \lambda=0.15408 \mathrm{~nm})$ at a scan rate of 4 degree/min. The morphology of the samples was characterized by a Scanning electron microscope (SEM) JSM-7001F, equipped with a QX200 energy dispersive X-ray spectrometer (EDS) for the chemical analysis. The transmission electron microscopy (TEM) was conducted utilizing a Tecnai G2 F20 S-Twin instrument.

The organic species contained in samples were characterized by Fourier trans form infrared (FTIR) using Bruker VERTEX 70 spectrometers with the wave length of $4000-400 \mathrm{~cm}^{-1}$ at resolution of $8 \mathrm{~cm}^{-1}$. The tablets were pressed with the mixture at a mass ratio of sample $\mathrm{KBr}=1: 400$. In situ diffusion Fourier transform infrared spectroscopy (DRIFTS) experiments were also performed on the same FTIR spectrometers. While the spectra in the range of $2000-1000 \mathrm{~cm}^{-1}$ was collected by accumulating 64 scans.

The redox ability of the samples was evaluated by $\mathrm{NO}+\mathrm{O}_{2}$-temperature programmed desorption (NO+O $\mathrm{O}_{2}$-TPD) using a Gasmet DX4000 FT-IR gas analyzer. Before $\mathrm{NO}$ and $\mathrm{O}_{2}$ co-adsorption, $0.1 \mathrm{~g}$ sample was pretreated with pure $\mathrm{N}_{2}$ at $300{ }^{\circ} \mathrm{C}$ (ramp rate, $10{ }^{\circ} \mathrm{C} / \mathrm{min}$ ) for $0.5 \mathrm{~h}$. Then the sample was cooled down to $50{ }^{\circ} \mathrm{C}$ and exposed to $400 \mathrm{ppm} \mathrm{NO}, 4.5$ vol \% $\mathrm{O}_{2}$ and $\mathrm{N}_{2}$ as balance gas $(300 \mathrm{~mL} / \mathrm{min})$ until adsorption equilibrium, followed by flushing the adsorbed sample with $\mathrm{N}_{2}$ for $2 \mathrm{~h}$ to remove physisorbed species. Finally, the $\mathrm{NO}_{2} / \mathrm{NO}$ desorption was carried out in $\mathrm{N}_{2}(30 \mathrm{~mL} / \mathrm{min})$ from $50{ }^{\circ} \mathrm{C}$ to $350{ }^{\circ} \mathrm{C}$ under a heating rate of $10{ }^{\circ} \mathrm{C} / \mathrm{min}$. The desorption amount of $\mathrm{NO}_{2} / \mathrm{NO}$ was measured by a thermal conductivity detector (TCD). The acid property and oxygen adsorption ability were also evaluated by $\mathrm{NH}_{3}$-TPD and $\mathrm{O}_{2}$-TPD, respectively. The processes were similar with that of $\mathrm{NO}+\mathrm{O}_{2}-\mathrm{TPD}$, except changing the reaction gas into $1000 \mathrm{ppm} \mathrm{NH}_{3}$ and pure $\mathrm{O}_{2}$. 
Table S1 Concentrations of previous investigations concerning N-doped carbons

\begin{tabular}{|c|c|c|c|}
\hline Carbonaceous material & $\begin{array}{l}\text { Nitrogen } \\
\text { contents }\end{array}$ & Modific ation conditions & reference \\
\hline $\begin{array}{l}\text { Nitrogen-rich porous } \\
\text { carbon }\end{array}$ & $9.56 \%$ & $\begin{array}{l}\text { in situ pyrolysis of sugar cane bagasse } \\
\text { with the mixture of urea, } \mathrm{ZnCl}_{2} \text { and } \\
\qquad \mathrm{KCl}\end{array}$ & Carbon 161 (2020) 25-35 \\
\hline $\begin{array}{c}\text { Nitrogen-doped } \\
\text { nanostructure carbon }\end{array}$ & $7.33 \%$ & $\begin{array}{l}\text { calcinated houttuynia was further } \\
\text { activated using different ratios of } \\
\mathrm{KOH} / \text { melamine }\end{array}$ & Carbon 161 (2020) 62-70 \\
\hline $\begin{array}{l}\text { Nitrogen enriched porous } \\
\text { carbons }\end{array}$ & $8.94 \%$ & $\begin{array}{l}\text { single-step co-hydrothermal treatment } \\
\text { of d-glucose and urea }\end{array}$ & $\begin{array}{c}\text { Chem. Eng. J. } 362 \text { (2019) } \\
\text { 794-801 }\end{array}$ \\
\hline $\begin{array}{l}\text { High-content } \mathrm{N} \text {-doped } \\
\text { CNT microspheres }\end{array}$ & $12.43 \%$ & $\begin{array}{l}\text { one-step annealing the } \mathrm{N} \text {-rich } \\
\text { HCl-treated melamine in metal ion } \\
\qquad\left(\mathrm{Ni}^{2+} / \mathrm{Co}^{2+} / \mathrm{Fe}^{3+}\right)\end{array}$ & $\begin{array}{l}\text { Adv. Funct. Mater. 29(39) } \\
\text { (2019) } 1904819\end{array}$ \\
\hline $\begin{array}{l}\text { Highly nitrogen doped } \\
\text { carbon nanofibers }\end{array}$ & $8.1 \%$ & annealing the polypyrrole nanofibers & $\begin{array}{l}\text { Nature communications } \\
\text { 9(1) (2018) } 1720\end{array}$ \\
\hline $\begin{array}{l}\text { nitrogen-rich porous } \\
\text { carbon nanosheets }\end{array}$ & $13.2 \%$ & $\begin{array}{l}\text { calcination the mixture of } \mathrm{g}-\mathrm{C}_{3} \mathrm{~N}_{4} \text { and } \\
\text { petroleum coke }\end{array}$ & $\begin{array}{c}\text { Journal of Materials } \\
\text { Chemistry A } 6.26 \text { (2018): } \\
\text { 12551-12558 }\end{array}$ \\
\hline $\begin{array}{l}\text { N-enriched carbon/carbon } \\
\text { nanotube composite }\end{array}$ & $17.53 \%$ & $\begin{array}{l}\text { in situ polymerization the melamine, } \\
\text { 1,4-Phthalaldehyde and multiw alled } \\
\text { carbon nanotube }\end{array}$ & $\begin{array}{l}\text { Small 14(12) (2018) } \\
1703569\end{array}$ \\
\hline $\begin{array}{l}\text { Nitrogen-enriched active } \\
\text { carbon fiber/reduced } \\
\text { graphene oxide } \\
\text { nanocomposite }\end{array}$ & $12 \%$ & $\begin{array}{l}\text { Carbonization the ACF film/GO/urea } \\
\text { composite }\end{array}$ & $\begin{array}{c}\text { Carbon } 130(2018) \\
196-205\end{array}$ \\
\hline $\begin{array}{l}\text { Nitrogen-doped porous } \\
\text { carbon nanosheets }\end{array}$ & $11.9 \%$ & $\begin{array}{c}\text { annealing the } C_{3} N_{4} \text { nanosheets and } \\
\text { PILs in a mass rate } 1: 10\end{array}$ & $\begin{array}{c}\text { Journal of Materials } \\
\text { Chemistry A 4(19) (2016) } \\
\text { 7313-7321 }\end{array}$ \\
\hline $\begin{array}{l}\text { Ultrahigh N-doped } \\
\text { carbon/graphene } \\
\text { sandwiched nanosheets }\end{array}$ & $15.4 \%$ & $\begin{array}{l}\text { annealing polypyrrole coated graphene } \\
\text { oxide nanocomposites }\end{array}$ & $\begin{array}{c}\text { Journal of Materials } \\
\text { Chemistry A 4(4) (2016) } \\
\text { 1423-1431 }\end{array}$ \\
\hline $\begin{array}{l}\text { High graphite N-doped } \\
\text { graphene }\end{array}$ & $8.83 \%$ & $\begin{array}{l}\text { one-step hydrothermal the graphene } \\
\text { oxide and urea with mass ratio 1:300 }\end{array}$ & $\begin{array}{l}\text { Green Chemistry } 18(15) \\
\text { (2016) } 4254-4262\end{array}$ \\
\hline $\begin{array}{l}\text { Pyridinic N-enriched } \\
\text { porous carbons }\end{array}$ & $17.71 \%$ & $\begin{array}{c}\text { one-step pyrolysis of raw rice husk, } \\
\mathrm{KOH} \text { and melamine with mass ratio } \\
1: 0.5: 3\end{array}$ & This work \\
\hline
\end{tabular}


Table S2 Concentration of nitrogen components in N1s spectra

\begin{tabular}{|c|c|c|c|c|c|c|c|c|c|}
\hline \multirow{2}{*}{ Samples } & \multirow{2}{*}{$\begin{array}{c}\text { Total } \mathrm{N} \\
\text { ratio }(\%)\end{array}$} & \multicolumn{2}{|c|}{ pyridinic $\mathrm{N}$} & \multicolumn{2}{|c|}{ Pyrrolic N } & \multicolumn{2}{|c|}{ graphitic $\mathrm{N}$} & \multicolumn{2}{|c|}{ oxidized N } \\
\hline & & $N-6^{a}$ & $\mathrm{~N}-6^{\mathrm{b}}$ & $\mathrm{N}-5^{\mathrm{a}}$ & $\mathrm{N}-5^{\mathrm{b}}$ & $\mathrm{N}-\mathrm{Q}^{\mathrm{a}}$ & $\mathrm{N}-\mathrm{Q}^{\mathrm{b}}$ & $\mathrm{N}-\mathrm{O}^{\mathrm{a}}$ & $\mathrm{N}-\mathrm{O}^{\mathrm{b}}$ \\
\hline $\mathrm{RH} / \mathrm{K} / \mathrm{M}$ & 17.71 & 51.3 & 9.09 & 39.8 & 7.04 & 8.9 & 1.58 & - & - \\
\hline RH/M & 17.01 & 47.5 & 8.07 & 43.9 & 7.46 & 5.35 & 0.91 & 3.3 & 0.57 \\
\hline RH-K/M & 11.26 & 38.9 & 4.38 & 54.3 & 6.11 & - & - & 6.8 & 0.77 \\
\hline RH-M & 6.74 & 25.7 & 1.73 & 47.4 & 3.19 & 18.58 & 1.25 & 8.3 & 0.57 \\
\hline $\mathrm{RH} / \mathrm{K}-\mathrm{M}$ & 3.99 & 24.3 & 0.97 & 52.8 & 2.11 & 19.7 & 0.79 & 3.2 & 0.13 \\
\hline RH-K-M & 2.62 & - & - & 39.2 & 1.03 & 33.3 & 0.87 & 27.5 & 0.72 \\
\hline
\end{tabular}

a relative percentage of nitrogen in the material, $b$ absolute percentage of nitrogen in the material 


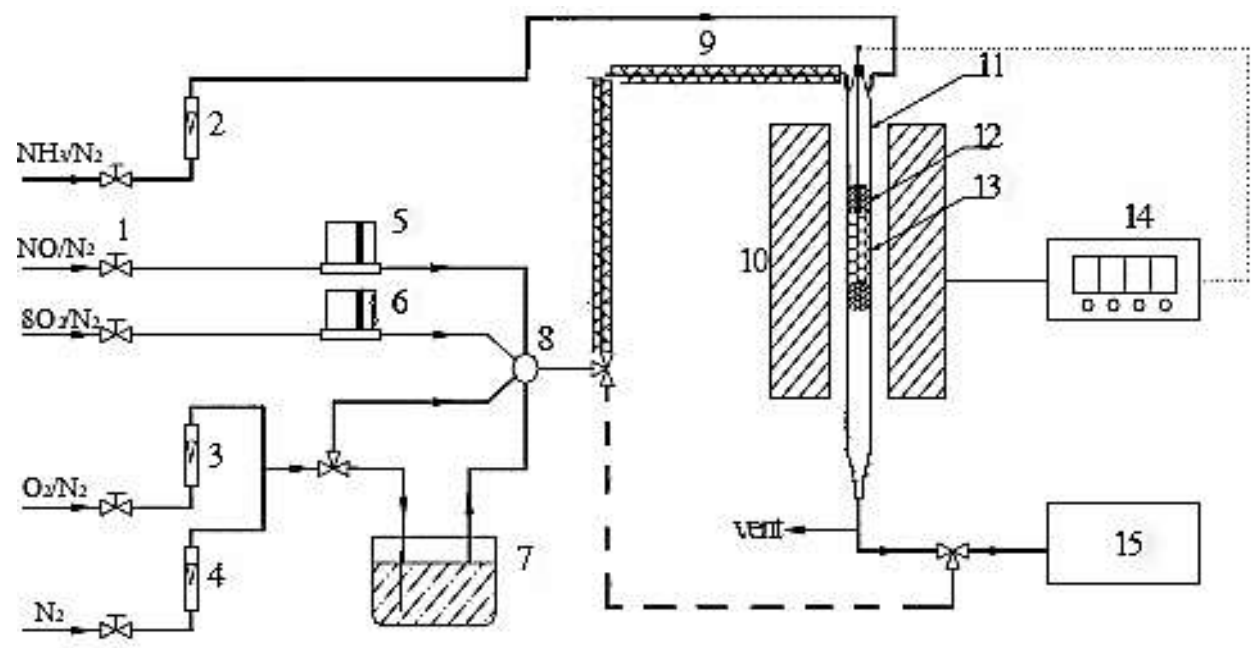

Figure S1. Schematic diagram of experimental apparatus for $\mathrm{NH}_{3}-\mathrm{SCR}$ reaction

1.flow rate controller; 2,3,4 rotameter; 5.6 mass flow controller; 7. water bath; 8. mixing chamber; 9 . preheater; 10. furnace; 11. reactor; 12. quartz wool; 13. catalysis; 14. program temperature controller; 15. FT-IR gas analyzer 


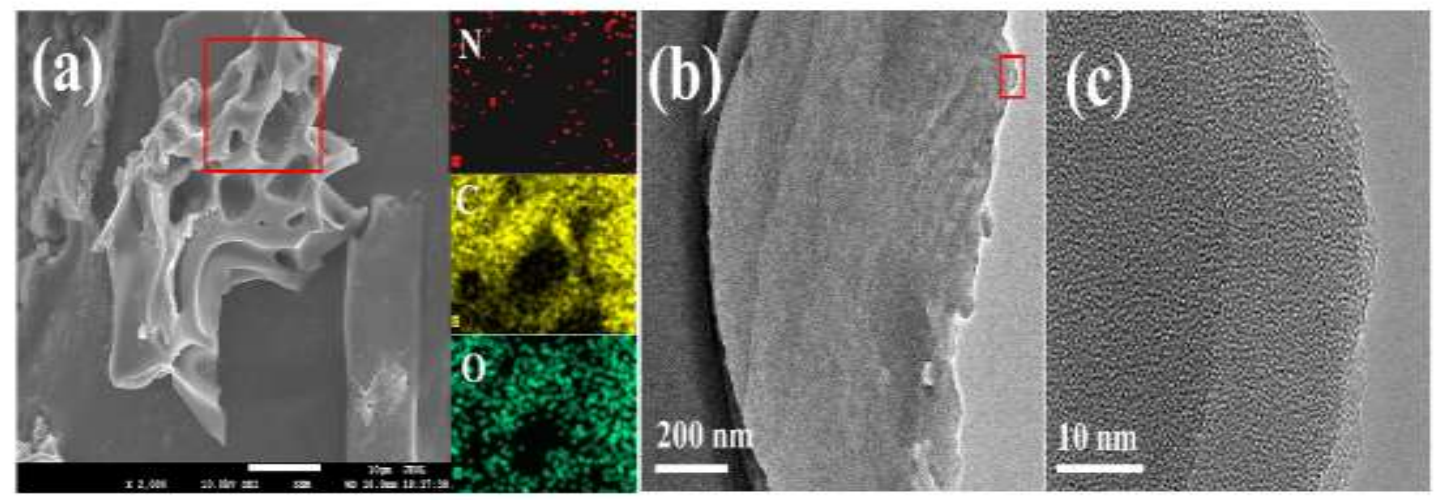

Figure S2. SEM images and corresponding elemental mapping (a), TEM (b) and HRTEM images (c) of rice husk. 


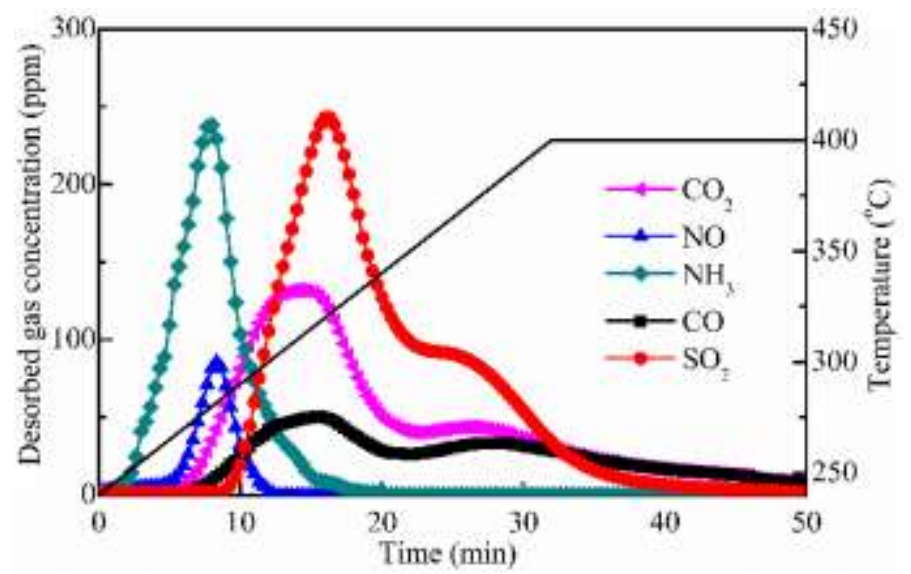

Figure S3. Gas desorption profile during regeneration 

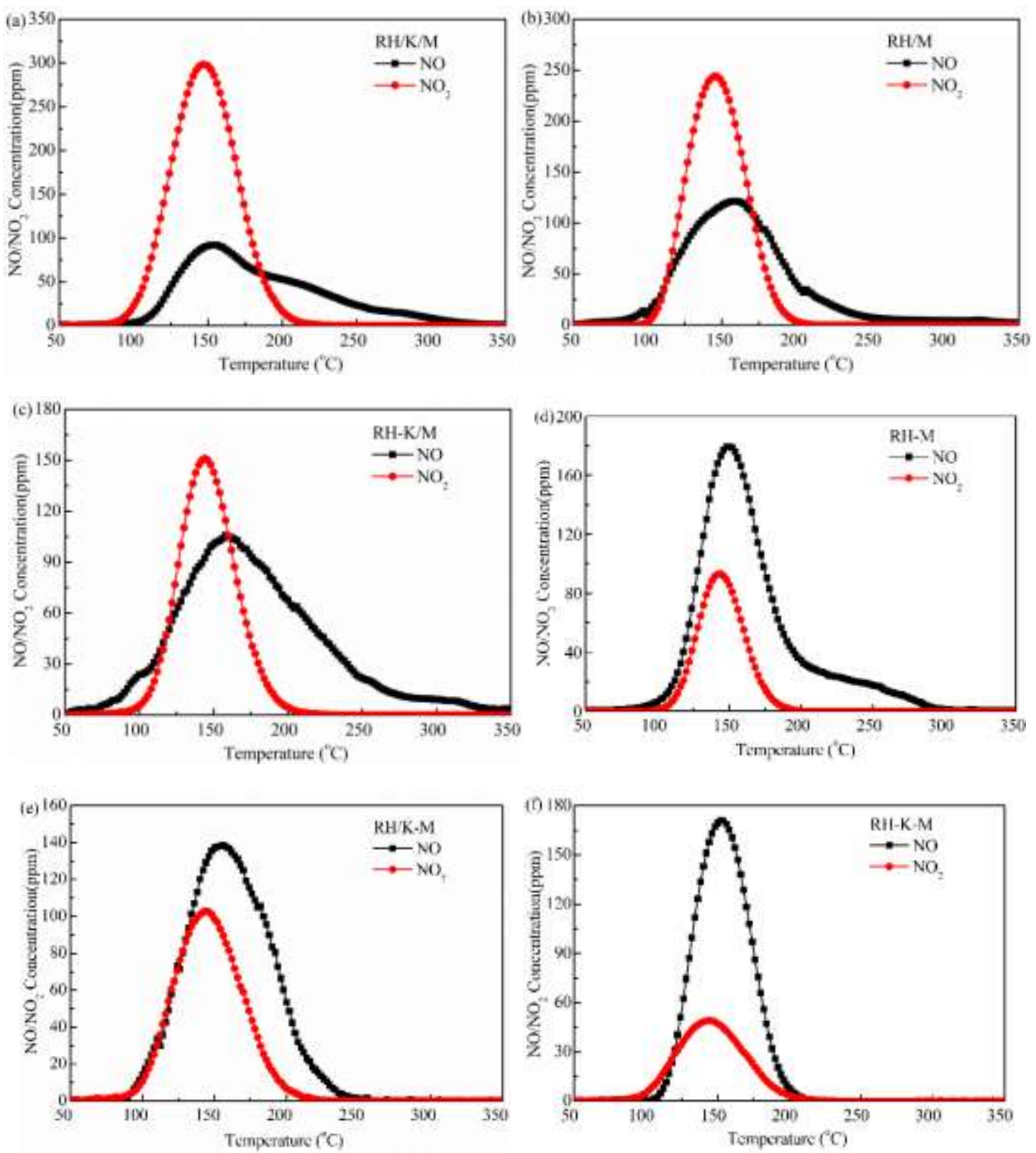

Figure S4. $\mathrm{NO} \mathrm{O}_{2}-\mathrm{TPD}$ profiles for $\mathrm{RH} / \mathrm{K} / \mathrm{M}$ (a); RH/M (b); RH-K/M (c); RH-M (d); RH/K-M (e); RH-K-M (f) 
Table S3 Concentration of desorbed $\mathrm{NO}$ and $\mathrm{NO}_{2}$

\begin{tabular}{ccccc}
\hline \multirow{2}{*}{ Samples } & \multicolumn{2}{c}{ Amounts of desorbed NOx (mmol/g) } & \multirow{2}{*}{$\mathrm{NO}_{2} / \mathrm{NO}_{\mathrm{X}}(\%)$} \\
\cline { 2 - 4 } & $\mathrm{NO}$ & $\mathrm{NO}_{2}$ & $\mathrm{NO}_{\mathrm{x}}$ & \\
\hline $\mathrm{RH} / \mathrm{K} / \mathrm{M}$ & 0.193 & 0.368 & 0.561 & 65.6 \\
$\mathrm{RH} / \mathrm{M}$ & 0.198 & 0.277 & 0.475 & 58.3 \\
$\mathrm{RH}-\mathrm{K} / \mathrm{M}$ & 0.162 & 0.146 & 0.308 & 47.4 \\
$\mathrm{RH}-\mathrm{M}$ & 0.139 & 0.067 & 0.206 & 32.5 \\
$\mathrm{RH} / \mathrm{K}-\mathrm{M}$ & 0.178 & 0.117 & 0.295 & 39.7 \\
$\mathrm{RH}-\mathrm{K}-\mathrm{M}$ & 0.127 & 0.045 & 0.172 & 26.2 \\
\hline
\end{tabular}

